\title{
Pointwise Analog of the Stečkin Approximation Theorem
}

\author{
Włodzimierz Lenski \\ Faculty of Mathematics, Computer Science and Econometrics, University of Zielona Góra, \\ Ulica Szafrana 4a, 65-516 Zielona Góra, Poland \\ Correspondence should be addressed to Włodzimierz Łenski; w.lenski@wmie.uz.zgora.pl \\ Received 11 November 2012; Accepted 20 January 2013 \\ Academic Editor: Roberto Renò
}

Copyright (C) 2013 Włodzimierz Łenski. This is an open access article distributed under the Creative Commons Attribution License, which permits unrestricted use, distribution, and reproduction in any medium, provided the original work is properly cited.

We show the pointwise version of the Stečkin theorem on approximation by de la Vallée-Poussin means. The result on norm approximation is also derived.

\section{Introduction}

Let $L^{p} \quad(1 \leq p<\infty) \quad[C]$ be the class of all $2 \pi$-periodic realvalued functions integrable in the Lebesgue sense with $p$ th power (continuous) over $Q=[-\pi, \pi]$, and let $X^{p}=L^{p}$ when $1 \leq p<\infty$ or $X^{p}=C$ when $p=\infty$.

Let us define the norms of $f \in X^{p}$ as

$$
\begin{aligned}
& \|f\|=\|f\|_{X^{p}}=\|f(\cdot)\|_{X^{p}} \\
& := \begin{cases}\left\{\int_{Q}|f(x)|^{p} d x\right\}^{1 / p} & \text { when } 1 \leq p<\infty, \\
\sup _{x \in Q}|f(x)| & \text { when } p=\infty,\end{cases} \\
& \|f\|_{x, \delta} \\
& =\|f\|_{X^{p}, x, \delta}=\|f(\cdot)\|_{X^{p}, x, \delta}:=\sup _{0<h \leq \delta}\|f(\cdot)\|_{X^{p}, x, h}^{\circ} \\
& := \begin{cases}\sup _{0<h \leq \delta}\left\{\frac{1}{2 h} \int_{x-h}^{x+h}|f(t)|^{p} d t\right\}^{1 / p} & \text { when } 1 \leq p<\infty, \\
\sup _{0<h \leq \delta}\left\{\sup _{0<|t| \leq h}|f(x+t)|\right\} & \text { when } p=\infty,\end{cases}
\end{aligned}
$$

where

$$
\begin{aligned}
& \|f\|_{x, \delta}^{\circ} \\
& =\|f\|_{X^{p}, x, \delta}^{\circ}=\|f(\cdot)\|_{X^{p}, x, \delta}^{\circ}
\end{aligned}
$$

$$
:= \begin{cases}\left\{\frac{1}{2 \delta} \int_{x-\delta}^{x+\delta}|f(t)|^{p} d t\right\}^{1 / p} & \text { when } 1 \leq p<\infty \\ \sup _{0<|h| \leq \delta}|f(x+h)| & \text { when } p=\infty\end{cases}
$$

We note additionally that

$$
\|f\|_{C, x, 0}=\|f\|_{C, x, 0}^{\circ}=|f(x)| .
$$

Consider the trigonometric Fourier series of $f$

$$
S f(x)=\frac{a_{o}(f)}{2}+\sum_{k=0}^{\infty}\left(a_{k}(f) \cos k x+b_{k}(f) \sin k x\right)
$$

with the partial sums $S_{k} f$.

Let

$$
\sigma_{n, m} f(x):=\frac{1}{m+1} \sum_{k=n-m}^{n} S_{k} f(x) \quad(m \leq n=0,1,2, \ldots) .
$$

As a measure of approximation by the previous quantities, we use the pointwise characteristics

$$
\begin{aligned}
& w_{x} f(\delta) \\
& =w_{x} f(\delta)_{X^{p}}:=\left\|\Delta_{x} f(\cdot)\right\|_{X^{p}, x, \delta}
\end{aligned}
$$




$$
= \begin{cases}\sup _{0<h \leq \delta}\left\{\frac{1}{2 h} \int_{-h}^{h}\left|\Delta_{x} f(t)\right|^{p} d t\right\}^{1 / p} & \text { when } 1 \leq p<\infty, \\ \sup _{0<h \leq \delta}\left\{\sup _{0<|t| \leq h}\left|\Delta_{x} f(t)\right|\right\} & \text { when } p=\infty,\end{cases}
$$

compared to [1], and

$$
\begin{aligned}
w_{x}^{\circ} f(\delta) & =w_{x}^{\circ} f(\delta)_{X^{p}}:=\left\|\Delta_{x} f(\cdot)\right\|_{X^{p}, x, \delta}^{0} \\
& = \begin{cases}\left\{\frac{1}{2 \delta} \int_{-\delta}^{\delta}\left|\Delta_{x} f(t)\right|^{p} d t\right\}^{1 / p} & \text { when } 1 \leq p<\infty, \\
\sup _{0<|t| \leq \delta}\left|\Delta_{x} f(t)\right| & \text { when } p=\infty,\end{cases}
\end{aligned}
$$

and also

$$
\begin{aligned}
& \Omega_{x} f\left(\frac{\pi}{n+1}\right)=\Omega_{x} f\left(\frac{\pi}{n+1}\right)_{X^{p}}:=\frac{1}{n+1} \sum_{k=0}^{n} w_{x} f\left(\frac{\pi}{k+1}\right)_{X^{p}}, \\
& \Omega_{x}^{\circ} f\left(\frac{\pi}{n+1}\right)=\Omega_{x}^{\circ} f\left(\frac{\pi}{n+1}\right)_{X^{p}}=\frac{1}{n+1} \sum_{k=0}^{n} w_{x}^{\circ} f\left(\frac{\pi}{k+1}\right)_{X^{p}}
\end{aligned}
$$

where

$$
\Delta_{x} f(t):=f(x+t)-f(x),
$$

constructed on the base of definition of $X^{p}$-points (Lebesgue points $\left(L^{p}\right.$-points) or points of continuity (C-points)). We also use the modulus of continuity of $f$ in the space $X^{p}$ defined by the formula

$$
\omega f(\delta)=\omega f(\delta)_{X^{p}}:=\sup _{0<|h| \leq \delta}\|\Delta . f(h)\|_{X^{p}}
$$

and its arithmetic mean

$$
\Omega f\left(\frac{\pi}{n+1}\right)=\Omega f\left(\frac{\pi}{n+1}\right)_{X^{p}}=\frac{1}{n+1} \sum_{k=0}^{n} \omega f\left(\frac{\pi}{k+1}\right)_{X^{p}} .
$$

We can observe that, for $f \in X^{\tilde{p}}$ and $\widetilde{p} \geq p$,

$$
\left\|w . f(\delta)_{X^{p}}\right\|_{C} \leq \omega f(\delta)_{C},
$$

whence

$$
\begin{gathered}
\left\|\Omega . f(\delta)_{X^{p}}\right\|_{C} \leq \Omega f(\delta)_{C}, \\
\left\|w_{.}^{\circ} f(\delta)_{X^{p}}\right\|_{X^{p}} \leq \omega f(\delta)_{X^{p}},
\end{gathered}
$$

whence

$$
\left\|\Omega_{.}^{\circ} f(\delta)_{X^{p}}\right\|_{X^{p}} \leq \Omega f(\delta)_{X^{p}} .
$$

Let us introduce one more measure of pointwise approximation analogical to the best approximation of function $f$ by trigonometric polynomials $T$ of the degree at most $n$ ( $T \in$ $\left.H_{n}\right)$

$$
E_{n}(f)_{X^{p}}:=\inf _{T \in H_{n}}\left\{\|f(\cdot)-T(\cdot)\|_{X^{p}}\right\} ;
$$

Namely,

$$
\begin{gathered}
E_{n}(f, x ; \delta) \\
=E_{n}(f, x ; \delta)_{X^{p}}:=\inf _{T \in H_{n}}\left\{\|f(\cdot)-T(\cdot)\|_{X^{p}, x, \delta}\right\} \\
=\left\{\begin{array}{c}
\inf _{T \in H_{n}}\left\{\operatorname { s u p } _ { 0 < h \leq \delta } \left[\frac{1}{2 h} \int_{-h}^{h} \mid f(x+t)\right.\right. \\
\left.\left.-\left.T(x+t)\right|^{p} d t\right]^{1 / p}\right\} \\
\text { when } 1 \leq p<\infty, \\
\inf _{T \in H_{n}}\left\{\sup _{0<|h| \leq \delta}|f(x+h)-T(x+h)|\right\} \\
\text { when } p=\infty,
\end{array}\right. \\
E_{n}^{\circ}(f, x ; \delta)=E_{n}^{\circ}(f, x ; \delta)_{X^{p}}:=\inf _{T \in H_{n}}\left\{\|f(\cdot)-T(\cdot)\|_{X^{p}, x, \delta}^{\circ}\right\} .
\end{gathered}
$$

We will also use its arithmetic mean

$$
\begin{aligned}
& F_{n, m}(f, x)=F_{n, m}(f, x)_{X^{p}}:=\frac{1}{m+1} \sum_{k=0}^{m} E_{n}\left(f, x ; \frac{\pi}{k+1}\right)_{X^{p}}, \\
& F_{n, m}^{\circ}(f, x)=F_{n, m}^{\circ}(f, x)_{X^{p}}:=\frac{1}{m+1} \sum_{k=0}^{m} E_{n}^{\circ}\left(f, x ; \frac{\pi}{k+1}\right)_{X^{p}} .
\end{aligned}
$$

Using these characteristics, we will show the pointwise version of the Stečkin [2] generalization of the Fejér-Lebesgue theorem. As a corollary, we will obtain the mentioned original result of Stečkin on norm approximation as well as the result of Tanović-Miller [3].

By $K$ we will designate either an absolute constant or a constant depending on some parameters, not necessarily the same of each occurrence.

\section{Statement of the Results}

At the beginning, we formulate the partial solution of the considered problem.

Theorem 1 (see [4]). If $f \in X^{p}$, then, for any positive integer $m \leq n$ and all real $x$, 


$$
\begin{aligned}
\left|\sigma_{n, m} f(x)-f(x)\right| \leq & \pi^{2} E_{n-m}^{\circ}\left(f, x, \frac{\pi}{2 n-m+1}\right)_{X^{p}} \\
& +6 F_{n-m, m}^{\circ}(f, x)_{X^{p}} \\
& +\int_{\pi /(2 n-m+1)}^{\pi /(m+1)} \frac{E_{n-m}^{\circ}(f, x, t)_{X^{p}}}{t} d t \\
& +E_{n-m}^{\circ}(f, x ; 0)_{X^{p}}, \\
\left|\sigma_{n, m} f(x)-f(x)\right| \leq & \left(6+\pi^{2}\right) F_{n-m, m}(f, x)_{X^{p}} \\
& \times\left[1+\ln \frac{n+1}{m+1}\right] \\
& +E_{n-m}(f, x ; 0)_{X^{p}} .
\end{aligned}
$$

Now, we can present the main result on pointwise approximation.

Theorem 2. If $f \in X^{p}$, then, for any positive integer $m \leq n$ and all real $x$,

$$
\begin{aligned}
& \left|\sigma_{n, m} f(x)-f(x)\right| \\
& \leq K \sum_{v=0}^{n} \frac{F_{n-m+v, m}(f, x)_{X^{p}}+F_{n-m+v, v}(f, x)_{X^{p}}}{m+v+1} \\
& \quad+E_{2 n}(f, x ; 0)_{X^{p}} .
\end{aligned}
$$

Remark 3. Theorem 2 , in case $f \in C$, immediately yields the result of Stečkin [2]. The result of Stečkin type also holds if instead of $C$ we consider the spaces $X^{p}$ with $1<p<\infty$. Then, in the proof, we need the Hardy-Littlewood estimate of the maximal function.

At every $X^{p}$-point $x$ of $f$

$$
\Omega_{x} f(\gamma)_{X^{p}}=o_{x}(1) \quad \text { as } \gamma \longrightarrow 0+
$$

and thus from Theorem 1, we obtain the corollary which states the result of the Tanović-Miller type [3].

Corollary 4. If $f \in X^{p}$, then, for any positive integer $m \leq n$ at every $X^{p}$-point $x$ of $f$,

$$
\left|\sigma_{n, m} f(x)-f(x)\right|=o_{x}(1)\left[1+\ln \frac{n+1}{m+1}\right] \quad \text { as } n \longrightarrow \infty .
$$

\section{Auxiliary Results}

In order to prove our theorems, we require some lemmas.

Lemma 5. If $T_{n}$ is the trigonometric polynomial of the degree at most $n$ of the best approximation of $f \in X^{p}$ with respect to the norm $\|\cdot\|_{X^{p}}$, then it is also the trigonometric polynomial of the degree at most $n$ of the best approximation of $f \in X^{p}$ with respect to the norm $\|\cdot\|_{X^{p}, x, \delta}$ for any $\delta \in[0, \pi]$.
Proof. From the inequalities

$$
\begin{aligned}
\left\|E_{n}(f, \cdot, \delta)_{X^{p}}\right\|_{X^{p}} & \geq\left\|E_{n}^{\circ}(f, \cdot, \delta)_{X^{p}}\right\|_{X^{p}} \\
& =\|\| f-T_{n, \delta}\left\|_{X^{p}, \delta}^{\circ}\right\|_{X^{p}}=\left\|f-T_{n, \delta}\right\|_{X^{p}} \\
& \geq\left\|f-T_{n}\right\|_{X^{p}}=E_{n}(f)_{X^{p}}, \\
\left\|E_{n}^{\circ}(f, \cdot, \delta)_{X^{p}}\right\|_{X^{p}} & \leq\|\| f-T_{n}\left\|_{X^{p}, \delta}^{\circ}\right\|_{X^{p}} \\
& =\left\|f-T_{n}\right\|_{X^{p}}=E_{n}(f)_{X^{p}},
\end{aligned}
$$

where $T_{n, \delta}$ and $T_{n}$ are the trigonometric polynomials of the degree at most $n$ of the best approximation of $f \in X^{p}$ with respect to the norms $\|\cdot\|_{X^{p}, x, \delta}^{\circ}$ and $\|\cdot\|_{X^{p}}$, respectively, we obtain relation

$$
\left\|f-T_{n, \delta}\right\|_{X^{p}}=\left\|f-T_{n}\right\|_{X^{p}}=E_{n}(f)_{X^{p}},
$$

whence $T_{n, \delta}=T_{n}$ for any $\delta \in[0, \pi]$ by uniqueness of the trigonometric polynomial of the degree at most $n$ of the best approximation of $f \in X^{p}$ with respect to the norm $\|\cdot\|_{X^{p}}$ (e.g., see [5] p. 96). We can also observe that for such $T_{n}$ and any $h \in[0, \delta]$,

$$
\begin{aligned}
\left\|f-T_{n}\right\|_{X^{p}, x, h}^{\circ} & =E_{n}^{\circ}(f, x, h)_{X^{p}} \leq E_{n}(f, x, \delta)_{X^{p}} \\
& \leq\left\|f-T_{n}\right\|_{X^{p}, x, \delta} .
\end{aligned}
$$

Hence,

$$
E_{n}(f, x, \delta)_{X p}=\left\|f-T_{n}\right\|_{X^{p}, x, \delta}
$$

and our proof is complete.

Lemma 6. If $n \in \mathbb{N}_{0}$ and $\delta>0$, then $E_{n}(f, x ; \delta)_{X^{p}}$ is nonincreasing function of $n$ and nondecreasing function of $\delta$. These imply that for $m, n \in \mathbb{N}$ the function $F_{n, m}(f, x)_{X^{p}}$ is nonincreasing function of $n$ and $m$ simultaneously.

Proof. The first part of our statement follows from the property of the norm $\|\cdot\|_{x, \delta}$ and supremum. The second part is a consequence of the calculation

$$
\begin{aligned}
\frac{F_{n, m+1}(f, x)_{X^{p}}}{F_{n, m}(f, x)_{X^{p}}} & =\frac{m+1}{m+2}\left(1+\frac{E_{n}(f, x ; \pi /(m+2))_{X^{p}}}{\sum_{k=0}^{m} E_{n}(f, x ; \pi /(k+1))_{X^{p}}}\right) \\
& \leq \frac{m+1}{m+2}\left(1+\frac{E_{n}(f, x ; \pi /(m+1))_{X^{p}}}{\sum_{k=0}^{m} E_{n}(f, x ; \pi /(m+1))_{X^{p}}}\right) \\
& =\frac{m+1}{m+2}\left(1+\frac{1}{m+1}\right)=1 .
\end{aligned}
$$

Lemma 7. Let $m, n, q \in \mathbb{N}_{0}$ such that $m \leq n$ and $q \geq m+1$. If $f \in X^{p}$, then

$$
\left|\sigma_{n+q, m} f(x)-\sigma_{n, m} f(x)\right| \leq K F_{n-m, m}(f, x)_{X^{p}} \sum_{v=0}^{q-1} \frac{1}{m+v+1} .
$$


Proof. It is clear that

$$
\begin{aligned}
\sigma_{n, m} f(x) & =\frac{1}{m+1} \sum_{k=n-m}^{n} \frac{1}{\pi} \int_{-\pi}^{\pi} f(x+t) D_{k}(t) d t \\
& =\frac{1}{\pi} \int_{-\pi}^{\pi} f(x+t) V_{n, m}(t) d t,
\end{aligned}
$$

where

$$
\begin{aligned}
V_{n, m}(t) & =\frac{1}{m+1} \sum_{k=n-m}^{n} D_{k}(t), \\
D_{k}(t) & =\frac{\sin ((2 k+1) t / 2)}{2 \sin (t / 2)} .
\end{aligned}
$$

Hence, by orthogonality of the trigonometric system,

$$
\begin{aligned}
& \sigma_{n+q, m} f(x)-\sigma_{n, m} f(x) \\
& =\frac{1}{\pi} \int_{-\pi}^{\pi}\left[f(x+t)-T_{n-m}(x+t)\right] \\
& \times\left(V_{n+q, m}(t)-V_{n, m}(t)\right) d t \\
& =\frac{1}{\pi(m+1)} \sum_{k=n-m}^{n} \int_{-\pi}^{\pi}\left[f(x+t)-T_{n-m}(x+t)\right] \\
& \times\left(D_{k+q}(t)-D_{k}(t)\right) d t \\
& =\frac{1}{\pi(m+1)} \\
& \times \sum_{k=n-m}^{n} \int_{-\pi}^{\pi}\left[f(x+t)-T_{n-m}(x+t)\right] \\
& \times \frac{\sin ((2 k+2 q+1) t / 2)-\sin ((2 k+1) t / 2)}{2 \sin (t / 2)} d t \\
& =\frac{1}{\pi(m+1)} \sum_{k=n-m}^{n} \int_{-\pi}^{\pi}\left[f(x+t)-T_{n-m}(x+t)\right] \\
& \times \frac{\sin (q t / 2) \cos ((2 k+q 1) t / 2)}{\sin (t / 2)} d t,
\end{aligned}
$$

with trigonometric polynomial $T_{n-m}$ of the degree at most $n-$ $m$ of the best approximation of $f$.

$$
\text { Using the notations }
$$

$$
\begin{gathered}
I_{1}=\left[-\frac{\pi}{q}, \frac{\pi}{q}\right], \quad I_{2}=\left[-\frac{\pi}{m+1},-\frac{\pi}{q}\right] \cup\left[\frac{\pi}{q}, \frac{\pi}{m+1}\right], \\
I_{3}=\left[-\pi,-\frac{\pi}{m+1}\right] \cup\left[\frac{\pi}{m+1}, \pi\right],
\end{gathered}
$$

we get

$$
\begin{aligned}
\sum= & \frac{1}{\pi(m+1)} \\
& \times \sum_{k=n-m}^{n}\left(\int_{I_{1}}+\int_{I_{2}}+\int_{I_{3}}\right) \\
& \times\left[f(x+t)-T_{n-m}(x+t)\right] \\
& \times \frac{\sin (q t / 2) \cos ((2 k+q+1) t / 2)}{\sin (t / 2)} d t \\
= & +\sum_{2}+\sum_{3}, \\
\sum \leq & \frac{1}{\pi(m+1)} \sum_{k=n-m}^{n} \int_{I_{1}}\left|f(x+t)-T_{n-m}(x+t)\right| q d t \\
= & \frac{q}{\pi} \int_{I_{1}}\left|f(x+t)-T_{n-m}(x+t)\right| d t \\
\leq & 2 E_{n-m}\left(f, x ; \frac{\pi}{q}\right)_{X^{p}} .
\end{aligned}
$$

We next evaluate the sums $\sum_{2}$ and $\sum_{3}$ using the partial integrating and Lemma 5. Thus,

$$
\begin{aligned}
\sum_{2} \leq & \int_{I_{2}} \frac{\left|f(x+t)-T_{n-m}(x+t)\right|}{t} d t \\
= & 2\left[\frac{1}{2 t} \int_{-t}^{t}\left|f(x+u)-T_{n-m}(x+u)\right| d u\right]_{t=\pi / q}^{t=\pi /(m+1)} \\
& +2 \int_{\pi / q}^{\pi /(m+1)} \frac{1}{t}\left[\frac{1}{2 t} \int_{-t}^{t}\left|f(x+u)-T_{n-m}(x+u)\right| d u\right] d t \\
\leq & 2 E_{n-m}\left(f, x ; \frac{\pi}{m+1}\right)_{X^{p}}+2 \int_{-t}^{t} \frac{1}{t} E_{n-m}(f, x ; t)_{X^{p}} d t \\
\leq & 4 E_{n-m}\left(f, x ; \frac{\pi}{m+1}\right)_{X^{p}}\left[1+\ln \frac{q}{m+1}\right] \\
\leq & 4 E_{n-m}\left(f, x ; \frac{\pi}{m+1}\right)_{X^{p}}\left[1+\sum_{v=0}^{q-1} \frac{1}{m+v+1}\right],
\end{aligned}
$$

$\sum_{3} \leq \frac{1}{m+1}$

$$
\begin{aligned}
& \times \int_{I_{3}} \frac{\left|f(x+t)-T_{n-m}(x+t)\right|}{t} \\
& \quad \times\left|\sum_{k=n-m}^{n} \cos \left(k t+\frac{q+1}{2} t\right)\right| d t
\end{aligned}
$$




$$
\begin{aligned}
& \leq \frac{1}{m+1} \\
& \times \int_{I_{3}} \frac{\left|f(x+t)-T_{n-m}(x+t)\right|}{t} \\
& \times\left|\frac{2 \sin ((n+1) t / 2) \cos ((2 n-m+q+1) t / 2)}{2 \sin (t / 2)}\right| d t \\
& \leq \frac{\pi}{m+1} \int_{I_{3}} \frac{\left|f(x+t)-T_{n-m}(x+t)\right|}{t^{2}} d t \\
& =\frac{\pi}{m+1}\left\{2 \left[\frac{1}{2 t} \int_{-t}^{t} \mid f(x+u)\right.\right. \\
& \left.-T_{n-m}(x+u) \mid d u\right]_{t=\pi /(m+1)}^{t=\pi} \\
& +4 \int_{\pi /(m+1)}^{\pi} \frac{1}{t^{2}}\left[\frac{1}{2 t} \int_{-t}^{t} \mid f(x+u)\right. \\
& \left.\left.-T_{n-m}(x+u) \mid d u\right] d t\right\} \\
& \leq \frac{\pi}{m+1}\left\{2 E_{n-m}(f, x ; \pi)_{X^{p}}\right. \\
& \left.+4 \int_{\pi /(m+1)}^{\pi} \frac{1}{t^{2}} E_{n-m}(f, x ; t)_{X^{p}} d t\right\} \\
& =\frac{2 \pi}{m+1}\left\{E_{n-m}(f, x ; \pi)_{X^{p}}\right. \\
& \left.+2 \int_{1}^{m+1} \frac{E_{n-m}(f, x ; \pi / u)_{X^{p}}}{\pi^{2} / u^{2}} \frac{\pi d u}{u^{2}}\right\} \\
& =\frac{2 \pi}{m+1}\left\{E_{n-m}(f, x ; \pi)_{X^{p}}\right. \\
& \left.+\frac{2}{\pi} \sum_{k=0}^{m-1} \int_{k+1}^{k+2} E_{n-m}\left(f, x ; \frac{\pi}{u}\right)_{X^{p}} d u\right\} \\
& =\frac{2 \pi}{m+1}\left\{E_{n-m}(f, x ; \pi)_{X^{p}}\right. \\
& \left.+\frac{2}{\pi} \sum_{k=0}^{m-1} E_{n-m}\left(f, x ; \frac{\pi}{k+1}\right)_{X^{p}}\right\} \\
& \leq \frac{2 \pi+2 / \pi}{m+1} \sum_{k=0}^{m-1} E_{n-m}\left(f, x ; \frac{\pi}{k+1}\right)_{X^{p}}
\end{aligned}
$$

which proves Lemma 7.

Before formulating the next lemmas, we define a new difference. Let $m, n \in \mathbb{N}_{0}$ and $m \leq n$. Denote that

$$
\tau_{n, m} f(x):=(m+1)\left\{\sigma_{n+m+1, m} f(x)-\sigma_{n, m} f(x)\right\} .
$$

Lemma 8. Let $m, n, \mu \in \mathbb{N}_{0}$ such that $2 \mu \leq m \leq n$. If $f \in X^{p}$, then

$$
\left|\tau_{n, m} f(x)-\tau_{n-\mu, m-\mu} f(x)\right| \leq K \mu F_{n-\mu+1, \mu-1}(f, x)_{X^{p}} \ln \frac{m}{\mu} .
$$

Proof. The proof follows by the method of Leindler [6]. Namely,

$$
\begin{aligned}
& \tau_{n, m} f(x)-\tau_{n-\mu, m-\mu} f(x)\left(\sum_{k=n+m-2 \mu+2}^{n+m+1}-2 \sum_{k=n-\mu+1}^{n}\right)\left[S_{k} f(x)-f(x)\right], \\
&\left|\tau_{n, m} f(x)-\tau_{n-\mu, m-\mu} f(x)\right| \\
& \leq\left|\left(\sum_{k=n+m-2 \mu+2}^{n+m-\mu+1}-\sum_{k=n-\mu+1}^{n}\right)\left[S_{k} f(x)-f(x)\right]\right| \\
&+\left|\left(\sum_{k=n+m-\mu+2}^{n+m+1}-\sum_{k=n-\mu+1}^{n}\right)\left[S_{k} f(x)-f(x)\right]\right| \\
&=\mu\left|\sigma_{n+m-\mu+1, \mu-1} f(x)-\sigma_{n, \mu-1} f(x)\right| \\
&+\mu\left|\sigma_{n+m+1, \mu-1} f(x)-\sigma_{n, \mu-1} f(x)\right| .
\end{aligned}
$$

By Lemma 6 , for $2 \mu \leq m$,

$$
\begin{aligned}
& \left|\tau_{n, m} f(x)-\tau_{n-\mu, m-\mu} f(x)\right| \\
& \leq K \mu F_{n-\mu+1, \mu-1}(f, x)_{X^{p}}\left[1+\ln \frac{(n-\mu+1)+\mu-1}{\mu}\right] \\
& \quad+K \mu F_{n-\mu+1, \mu-1}(f, x)_{X^{p}}\left[1+\ln \frac{m+\mu-1}{\mu}\right] \\
& \quad \leq K \mu F_{n-\mu+1, \mu-1}(f, x)_{X^{p}}\left[1+\ln \frac{m}{\mu}\right],
\end{aligned}
$$

and our proof is complete.

Lemma 9. Let $m, n \in \mathbb{N}_{0}$ and $m \leq n$. If $f \in X^{p}$, then

$$
\left|\tau_{n, m} f(x)\right| \leq K \sum_{k=n-m}^{n} F_{k, k-n+m}(f, x)_{X^{p}} .
$$

Proof. Our proof runs parallel with the proof of Theorem 1 in [2].

$$
\text { If } m=0 \text {, then }
$$

$$
\left|\tau_{n, 0} f(x)\right|=\left|\sigma_{n+1,0} f(x)-\sigma_{n, 0} f(x)\right| \leq K F_{n, 0}(f, x)_{X^{p}},
$$

and if $m=1$, then

$$
\begin{aligned}
\left|\tau_{n, 1} f(x)\right| & \leq 2\left|\sigma_{n+2,1} f(x)-\sigma_{n, 1} f(x)\right| \\
& \leq K F_{n-1,1}(f, x)_{X^{p}}
\end{aligned}
$$




$$
\begin{aligned}
& \leq K\left[F_{n-1,1}(f, x)_{X^{p}}+F_{n-1,1}(f, x)_{X^{p}}\right] \\
& \leq K\left[F_{n-1,1}(f, x)_{X^{p}}+F_{n, 1}(f, x)_{X^{p}}\right]
\end{aligned}
$$

by Lemmas 6 and 7 .

Next, we construct the same decreasing sequence $\left(m_{s}\right)$ of integers that was given by Stečkin. Let

$$
m_{0}=m, \quad m_{s}=m_{s-1}-\left[\frac{m_{s-1}}{2}\right] \quad(s=1,2, \ldots),
$$

where $[y]$ denotes the integral part of $y$. It is clear that there exists smallest index $t \geq 1$ such that $m_{t}=1$ and

$$
m=m_{0}>m_{1}>\cdots>m_{t}=1 \text {. }
$$

By the definition of the numbers $m_{s}$, we have

$$
\begin{gathered}
m_{s} \geq \frac{m_{s-1}}{2}, \\
m_{s-1}-m_{s}=\left[\frac{m_{s-1}}{2}\right] \geq\left[\frac{m_{s-1}}{3}\right] \quad(s=1,2, \ldots, t)
\end{gathered}
$$

whence

$$
\begin{gathered}
m_{t-1}=2, \quad m_{t-1}-m_{t}=1, \\
m_{s-1}-m_{s} \leq m_{s} \leq 3\left(m_{s}-m_{s+1}\right) \quad(s=1,2, \ldots, t-1)
\end{gathered}
$$

follow.

Under these notations, we get the following equality:

$$
\begin{aligned}
\tau_{n, m} f(x)= & \sum_{s=1}^{t}\left(\tau_{n-m+m_{s-1}, m_{s-1}} f(x)-\tau_{n-m+m_{s}, m_{s}} f(x)\right) \\
& +\tau_{n-m+m_{t}, m_{t}} f(x),
\end{aligned}
$$

whence, by $m_{t}=1$,

$$
\begin{aligned}
\left|\tau_{n, m} f(x)\right| \leq & \sum_{s=1}^{t}\left|\tau_{n-m+m_{s-1}, m_{s-1}} f(x)-\tau_{n-m+m_{s}, m_{s}} f(x)\right| \\
& +\left|\tau_{n-m+m_{t}, m_{t}} f(x)\right|
\end{aligned}
$$

follows

It is easy to see that the terms in the sum $\sum_{s=1}^{t}$, by Lemma 8 , with $\mu=m_{s-1}-m_{s}$ and $m=m_{s-1}$ do not exceed

$$
\begin{array}{r}
K\left(m_{s-1}-m_{s}\right) F_{n-m+m_{s}+1, m_{s-1}-m_{s}}(f, x)_{X^{p}} \ln \frac{m_{s-1}}{m_{s-1}-m_{s}}, \\
\text { where }(s=1,2, \ldots, t-1),
\end{array}
$$

and by Lemma 7, we get

$$
\begin{aligned}
\left|\tau_{n-m+1,1} f(x)\right| & \leq 2\left|\sigma_{n-m+2,1} f(x)-\sigma_{n-m+1,1} f(x)\right| \\
& \leq K F_{n-m, 1}(f, x)_{X^{p}} .
\end{aligned}
$$

Thus,

$$
\begin{aligned}
\left|\tau_{n, m} f(x)\right| \leq & K \sum_{s=1}^{t-1} 3\left(m_{s}-m_{s+1}\right) F_{n-m+m_{s}+1, m_{s}}(f, x)_{X^{p}} \ln 3 \\
& +K F_{n-m+2, m-2}(f, x)_{X^{p}}+K F_{n-m, 1}(f, x)_{X^{p}}
\end{aligned}
$$

whence, by the monotonicity of $F_{v, \mu}(f, x)_{X^{p}}$,

$$
\begin{aligned}
& \left|\tau_{n, m} f(x)\right| \\
& \leq K\left(\sum_{s=1}^{t-1} \sum_{v=m_{s+1}+1}^{m_{s}} F_{n-m+v+1, v}(f, x)_{X^{p}}\right. \\
& \left.\quad+\sum_{v=0}^{2} F_{n-m+v, m-v-1}(f, x)_{X^{p}}\right) \\
& \quad+K F_{n-m, 1}(f, x)_{X^{p}} \\
& \leq K \sum_{v=0}^{m_{1}+1} F_{n-m+v, v}(f, x)_{X^{p}}+K F_{n-m, 1}(f, x)_{X^{p}} \\
& \leq K \sum_{v=0}^{m} F_{n-m+v, v}(f, x)_{X^{p}}+K F_{n-m, 1}(f, x)_{X^{p}} \\
& \leq K \sum_{k=n-m}^{n} F_{k, k-n+m}(f, x)_{X^{p}}+K F_{n-m, 1}(f, x)_{X^{p}} .
\end{aligned}
$$

\section{Proofs of the Results}

Proof of Theorem 2. The proof follows the lines of the proofs of Theorem 4 in [2] and Theorem in [6]. Therefore, let $n>0$ and $m \leq n$ be fixed. Let us define an increasing sequence $\left(n_{s}: s=0,1, \ldots, t\right)$ of indices introduced by Stečkin in the following way. Set $n_{0}=n$. Assuming that the numbers $n_{0}, \ldots, n_{s}$ are already defined and $n_{s}<2 n$, we define $n_{s+1}$ as follows. Let $v_{s}$ denote the smallest natural number such that

$$
F_{n_{s}-m+v_{s}, v}(f, x)_{X^{p}} \leq \frac{1}{2} F_{n_{s}-m, v}(f, x)_{X^{p}} \quad(v=0,1, \ldots, n) .
$$

According to the magnitude of $v_{s}$, we define

$$
n_{s+1}= \begin{cases}n_{s}-m+1 & \text { for } v_{s} \leq m, \\ n_{s}+v_{s} & \text { for } m+1 \leq v_{s}<2 n+m-n_{s}, \\ 2 n+m & \text { for } v_{s} \geq 2 n+m-n_{s} .\end{cases}
$$

If $n_{s+1}<2 n$, we continue the procedure, and if once $n_{s+1} \geq 2 n$, then we stop the construction and define $t:=s+1$.

By the previous definition of $\left(n_{s}\right)$, we have the following obvious properties:

$$
\begin{gathered}
t \geq 1, \quad n=n_{0}<n_{1}<\cdots<n_{t}, \quad 2 n \leq n_{t} \leq 2 n+m, \\
n_{s+1}-n_{s} \geq m+1 \quad(s=0,1, \ldots, t-1),
\end{gathered}
$$


and relations

$$
\begin{array}{r}
F_{n_{s+1}-m, v}(f, x)_{X^{p}} \leq \frac{1}{2} F_{n_{s}-m, v}(f, x)_{X^{p}} \\
\text { for } s=0,1, \ldots, t-2, \\
\frac{1}{2} F_{n_{s}-m, v}(f, x)_{X^{p}} \leq F_{n_{s+1}-m-1, v}(f, x)_{X^{p}} \\
\text { for } s=0,1, \ldots t-1,
\end{array}
$$

whenever $n_{s+1}-n_{s}>m+1$.

Let us start with

$$
\begin{aligned}
\mid \sigma_{n, m} f & (x)-f(x) \mid \\
= & \sum_{s=0}^{t-1}\left[\left|\sigma_{n_{s}, m} f(x)-f(x)\right|-\left|\sigma_{n_{s+1}, m} f(x)-f(x)\right|\right] \\
& +\left|\sigma_{n_{t}, m} f(x)-f(x)\right| \\
\leq & \sum_{s=0}^{t-1}\left|\sigma_{n_{s+1}, m} f(x)-\sigma_{n_{s}, m} f(x)\right|+\left|\sigma_{n_{t}, m} f(x)-f(x)\right| \\
= & \sum_{s=0}^{t-1}\left|\frac{1}{m+1} \tau_{n_{s}, m} f(x)\right|+\left|\sigma_{n_{t}, m} f(x)-f(x)\right| .
\end{aligned}
$$

Using Theorem 1 and that $2 n \leq n_{t} \leq 2 n+m$, we get

$$
\begin{aligned}
\mid \sigma_{n_{t}, m} f & (x)-f(x) \mid \\
\leq & K F_{n_{t}-m, m}(f, x)_{X^{p}}\left[1+\ln \frac{n_{t}+1}{m+1}\right] \\
& +\left|f(x)-T_{n_{t}-m}(x)\right| \\
\leq & K \sum_{v=0}^{n} \frac{F_{n-m+v, m}(f, x)_{X^{p}}}{m+v+1}+\left|f(x)-T_{n_{t}-m}(x)\right| \\
\leq & K \sum_{v=0}^{n} \frac{F_{n-m+v, m}(f, x)_{X^{p}}+F_{n-m+v, v}(f, x)_{X^{p}}}{m+v+1} \\
& +\left|f(x)-T_{n_{t}-m}(x)\right| \\
\leq & K \sum_{v=0}^{n} \frac{F_{n-m+v, m}(f, x)_{X^{p}}+F_{n-m+v, v}(f, x)_{X^{p}}}{m+v+1} \\
& +E_{n_{t}-m}(f, x ; 0)_{X^{p}} \\
\leq & K \sum_{v=0}^{n} \frac{F_{n-m+v, m}(f, x)_{X^{p}}+F_{n-m+v, v}(f, x)_{X^{p}}}{m+v+1} \\
& +E_{2 n}(f, x ; 0)_{X^{p}} .
\end{aligned}
$$

The estimate of the sum in the right hand side of (55) we derive from the following one

$$
\begin{aligned}
& \left|\frac{1}{m+1} \tau_{n_{s}, m} f(x)\right| \\
& \quad \leq K \sum_{v=0}^{n_{s+1}-n_{s}-1} \frac{F_{n_{s}-m+v, m}(f, x)_{X^{p}}+F_{n_{s}-m+v, m}(f, x)_{X^{p}}}{m+v+1} .
\end{aligned}
$$

We split the proof of this inequality in two parts. If $n_{s+1}-n_{s}=$ $m+1$, then by Lemma 9 ,

$$
\begin{aligned}
\left|\frac{1}{m+1} \tau_{n_{s}, m} f(x)\right| & \leq K \frac{1}{m+1} \sum_{k=n_{s}-m}^{n_{s}} F_{k, k-n_{s}+m}(f, x)_{X^{p}} \\
& \leq K \sum_{v=0}^{n_{s+1}-n_{s}-1} \frac{F_{n_{s}-m+v, v}(f, x)_{X^{p}}}{m+v+1} .
\end{aligned}
$$

If $n_{s+1}-n_{s}>m+1$, then, by Lemma 7 ,

$$
\begin{aligned}
& \left|\frac{1}{m+1} \tau_{n_{s}, m} f(x)\right| \\
& \quad \leq K F_{n_{s}-m, m}(f, x)_{X^{p}} \sum_{v=0}^{n_{s+1}-n_{s}-1} \frac{1}{m+v+1},
\end{aligned}
$$

and since $(1 / 2) F_{n_{s}-m, m}(f, x)_{X^{p}} \leq F_{n_{s+1}-m-1, m}(f, x)_{X^{p}}$, we have

$$
\begin{aligned}
& \left|\frac{1}{m+1} \tau_{n_{s}, m} f(x)\right| \\
& \quad \leq 2 K F_{n_{s+1}-m-1, m}(f, x)_{X^{p}} \sum_{v=0}^{n_{s+1}-n_{s}-1} \frac{1}{m+v+1} \\
& \quad \leq 2 K \sum_{v=0}^{n_{s+1}-n_{s}-1} \frac{F_{n_{s}-m+v, m}(f, x)_{X^{p}}}{m+v+1} .
\end{aligned}
$$

Consequently,

$$
\begin{aligned}
& \sum_{s=0}^{t-1}\left|\frac{1}{m+1} \tau_{n_{s}, m} f(x)\right| \\
& \quad \leq 2 K \sum_{s=0}^{t-1} \sum_{v=0}^{n_{s+1}-n_{s}-1} \frac{F_{n_{s}-m+v, m}(f, x)_{X^{p}}+F_{n_{s}-m+v, v}(f, x)_{X^{p}}}{m+v+1} .
\end{aligned}
$$

Since $n_{s+1}-n_{s} \leq 2 n+m-n-1=n+m-1$ for all $s \leq t-1$, changing the order of summation, we get

$$
\begin{aligned}
& \sum_{s=0}^{t-1} \mid \frac{1}{m+1} \tau_{n_{s}, m} f(x) \mid \\
& \leq 2 K \sum_{v=0}^{n+m-1} \frac{1}{m+v+1} \\
& \quad \times \sum_{s: n_{s+1}-n_{s}>v}\left[F_{n_{s}-m+v, m}(f, x)_{X^{p}}+F_{n_{s}-m+v, v}(f, x)_{X^{p}}\right] .
\end{aligned}
$$


Using the inequality

$$
\begin{aligned}
& F_{n_{s+1}-m, v}(f, x)_{X^{p}} \leq \frac{1}{2} F_{n_{s}-m, v}(f, x)_{X^{p}} \\
& \text { for }\left\{\begin{array}{l}
v=0,1,2, \ldots, n_{s+1}-n_{s}-1, \\
s=0,1,2, \ldots, t-2,
\end{array}\right.
\end{aligned}
$$

we obtain

$$
\begin{aligned}
\sum_{s: n_{s+1}-n_{s}>v} & {\left[F_{n_{s}-m+v, m}(f, x)_{X^{p}}+F_{n_{s}-m+v, v}(f, x)_{X^{p}}\right] } \\
= & F_{n_{p}-m+v, m}(f, x)_{X^{p}}+F_{n_{p}-m+v, v}(f, x)_{X^{p}} \\
& +\sum_{s \geq p+1: n_{s+1}-n_{s}>v}\left[F_{n_{s+1}-m+v, m}(f, x)_{X^{p}}\right. \\
& \left.+F_{n_{s+1}-m+v, v}(f, x)_{X^{p}}\right] \\
\leq & F_{n_{p}-m+v, m}(f, x)_{X^{p}}+F_{n_{p}-m+v, v}(f, x)_{X^{p}} \\
& +\sum_{s: s \geq p+1} F_{n_{s}-m, m}(f, x)_{X^{p}}\left[F_{n_{s}-m, m}(f, x)_{X^{p}}\right. \\
\leq & F_{n_{p}-m+v, m}(f, x)_{X^{p}}+F_{n_{p}-m+v, v}(f, x)_{X^{p}} \\
& +2\left[F_{n_{p+1}-m, m}(f, x)_{X^{p}}+F_{n_{p+1}-m, v}(f, x)_{X^{p}}\right] \\
\leq & 3\left[F_{n_{p}-m+v, m}(f, x)_{X^{p}}+F_{n_{p}-m+v, v}(f, x)_{X^{p}}\right],
\end{aligned}
$$

where $p$ denotes the smallest index $s$ having the property $n_{s+1}-n_{s}>v$. Hence,

$$
\begin{aligned}
& \sum_{s=0}^{t-1}\left|\frac{1}{m+1} \tau_{n_{s}, m} f(x)\right| \\
& \quad \leq K \sum_{v=0}^{n+m-1} \frac{F_{n-m+v, m}(f, x)_{X^{p}}+F_{n-m+v, v}(f, x)_{X^{p}}}{m+v+1} \\
& \quad \leq K \sum_{v=0}^{n} \frac{F_{n-m+v, m}(f, x)_{X^{p}}+F_{n-m+v, v}(f, x)_{X^{p}}}{m+v+1}
\end{aligned}
$$

and our proof follows.

\section{References}

[1] S. Aljančić, R. Bojanić, and M. Tomić, "On the degree of convergence of Fejéer-Lebesgue sums," L'Enseignement Mathématique. Revue Internationale. IIe Série, vol. 15, pp. 21-28, 1969.

[2] S. B. Stečkin, "On the approximation of periodic functions by de la Vallée Poussin sums," Analysis Mathematica, vol. 4, no. 1, pp. 61-74, 1978.

[3] N. Tanović-Miller, "On some generalizations of the FejérLebesgue theorem," Unione Matematica Italiana. Bollettino. B. Serie 6, vol. 1, no. 3, pp. 1217-1233, 1982.

[4] W. Łenski, "Pointwise approximation by de la Vallée-Poussin means," East Journal on Approximations, vol. 14, no. 2, pp. 131136, 2008.
[5] P. L. Butzer and R. J. Nessel, Fourier Analysis and Approximation, Academic Press, New York, NY, USA, 1971.

[6] L. Leindler, "Sharpening of Stečkin's theorem to strong approximation," Analysis Mathematica, vol. 16, no. 1, pp. 27-38, 1990. 


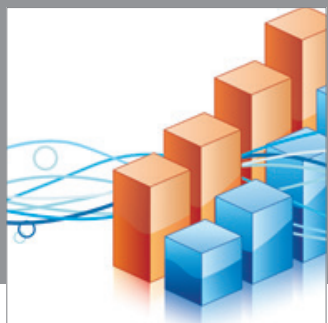

Advances in

Operations Research

mansans

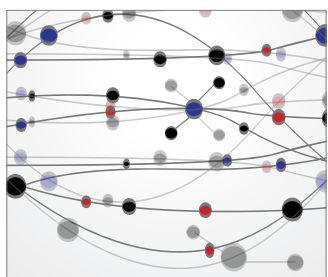

The Scientific World Journal
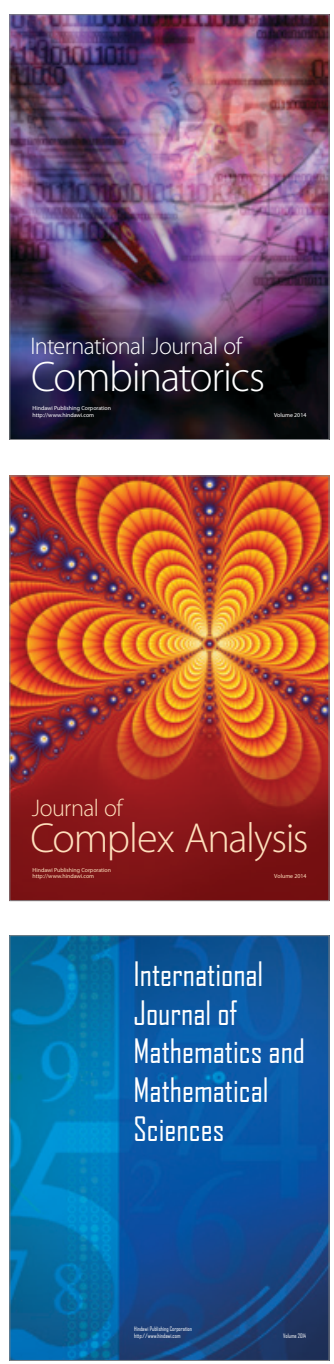
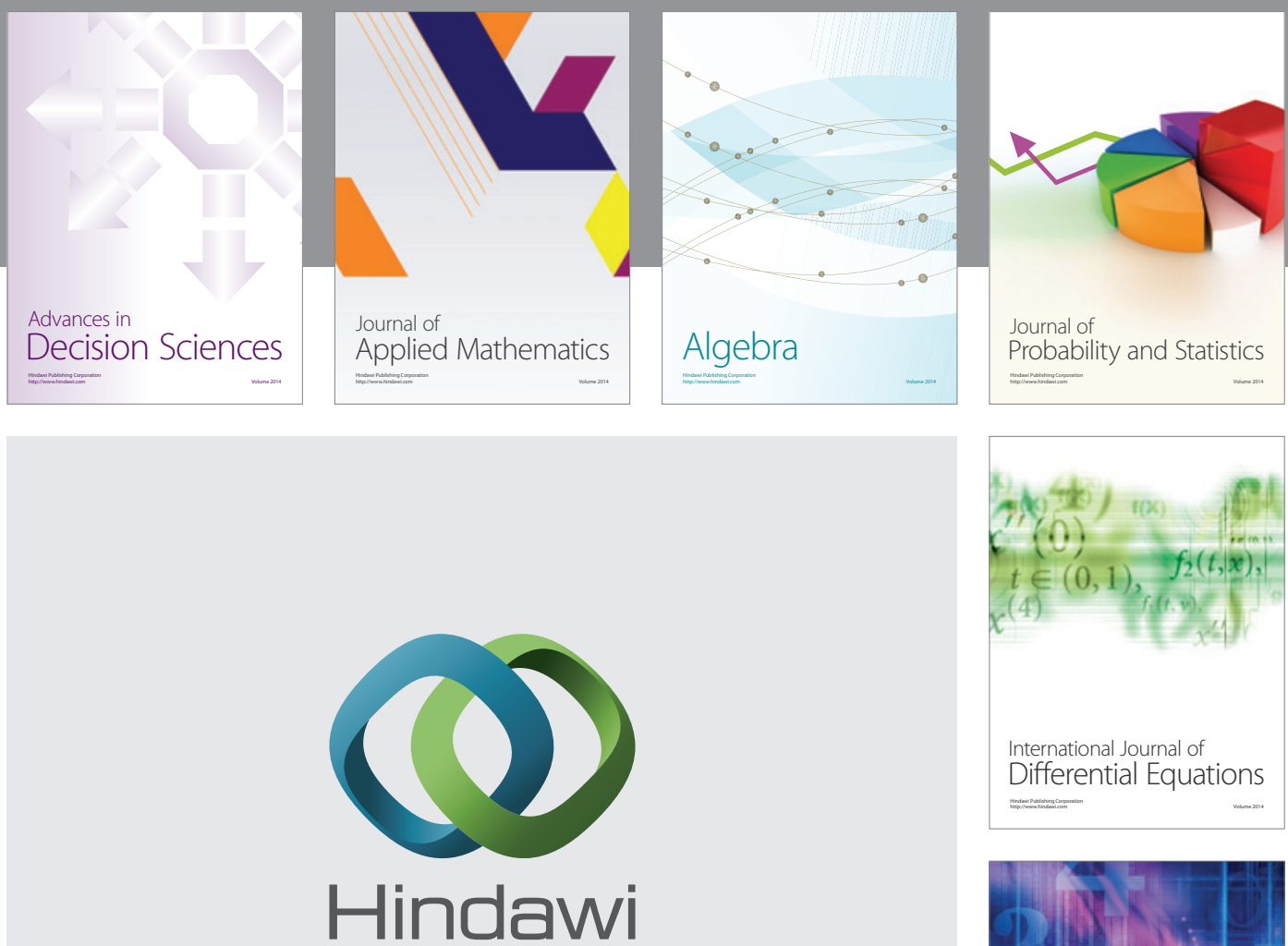

Submit your manuscripts at http://www.hindawi.com
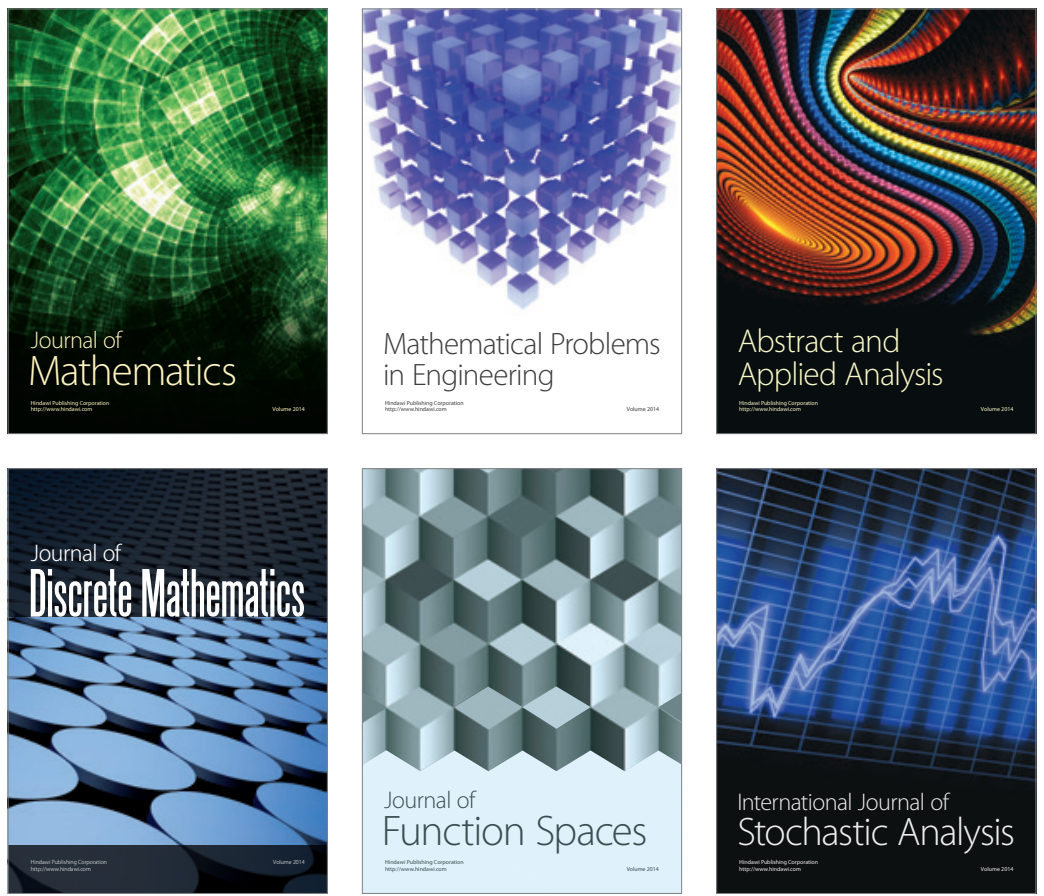

Journal of

Function Spaces

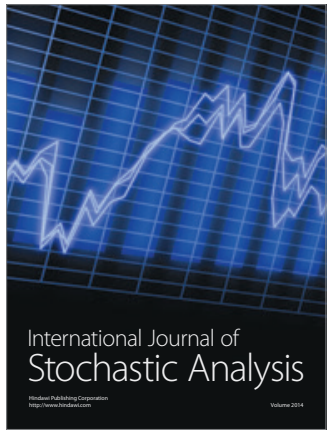

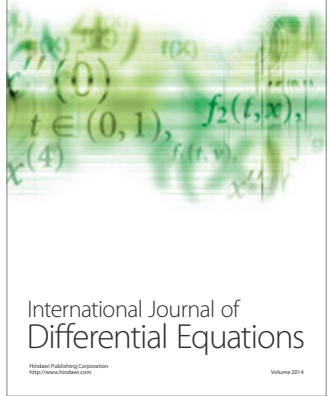
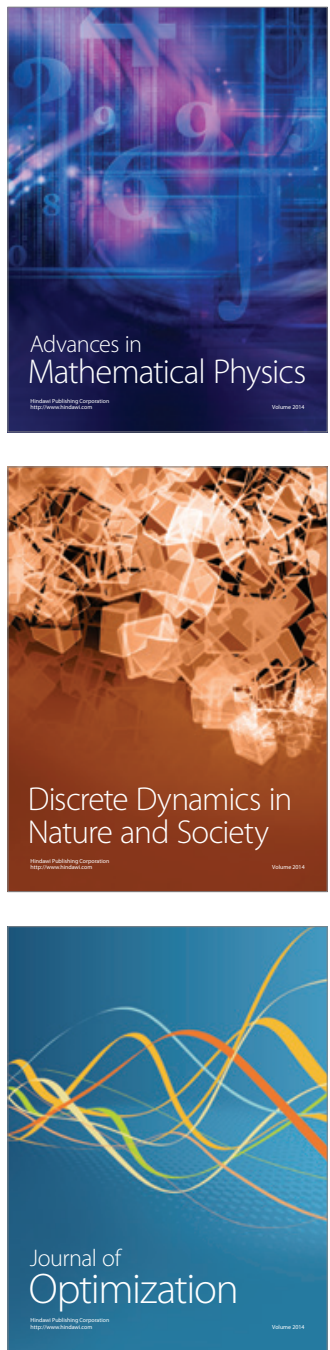